\title{
DIAGNOSTIC APPROACH TO ABDOMINAL PAIN IN SCHOOL-AGE CHILDREN
}

\author{
Tudor Lucian Pop \\ $2^{\text {nd }}$ Pediatric Discipline, Mother and Child Department, "Iuliu Hațieganu" University of Medicine and \\ Pharmacy Cluj-Napoca, Romania \\ $2^{\text {nd }}$ Pediatric Clinic, Emergency Clinical Hospital for Children Cluj-Napoca, Romania
}

\begin{abstract}
:
Abdominal pain is probably the most common cause of addressing a doctor or of school absenteeism. The family doctor, the pediatrician, and the school doctor must identify the red flags that can direct the diagnosis towards an organic cause (acute, chronic, or even a surgical emergency). They also have to be aware of functional causes of abdominal pain in school-age children and adolescents. The pathogenesis of functional abdominal pain is multifactorial, based on the interrelationship between visceral hypersensitivity and the alteration of gastrointestinal motility. The Roma IV criteria help a proactive diagnosis of functional gastrointestinal disorders: functional dyspepsia, irritable bowel syndrome, abdominal migraine, and functional abdominal pain. In functional abdominal pain, there are no biochemical markers or structural changes that allow an objective diagnosis or the monitoring of these disorders. The decision to use diagnostic tests is left to the physician. Functional abdominal pain can influence a child's school performance and social relationships and may associate anxiety, depression, and extra-intestinal manifestations, with poor quality of life. Thus a correct diagnosis and management of abdominal pain are essential for these children.
\end{abstract}

Keywords: abdominal pain, functional, Roma criteria, child, adolescent

Abdominal pain in children is probably the most common cause for seeing a doctor or for school absenteeism. Although pain is a symptom of a benign condition in many cases, it is of great importance to properly evaluate it to eliminate possible causes of emergency, including surgical emergencies. The family doctor, the pediatrician, and the school doctor must identify the red flags that can direct the diagnosis towards an organic cause (acute, chronic) and have knowledge about the functional causes of abdominal pain in school-age children and adolescents.

Acute abdominal pain accounts for about $9 \%$ of children's visits to the primary care physician (family doctor or school physician). Most of the time, the pathology responsible for the pain is not an emergency. Still, the doctor's most difficult task is to make a correct diagnosis, especially in cases involving surgical emergencies. The incidence of the acute surgical abdomen is described to be $2 \%$. [1,2]

Acute abdominal pain can have different causes, some more common in early childhood, others more common in adolescence. Table 1 contains the most common etiologies of acute abdominal pain depending on the age of the child.

\footnotetext{
* Corresponding author: Tudor Lucian Pop, $2^{\text {nd }}$ Pediatric Discipline, Mother and Child Department, "Iuliu Hațieganu" University of Medicine and Pharmacy Cluj-Napoca, Romania, ${ }^{\text {nd }}$ Pediatric Clinic, Emergency Clinical Hospital for Children Cluj-Napoca, Romania, e-mail: tudorlpop@yahoo.com
}

Article received: 04.04.2021, accepted: 09.04.2021, published: 12.04.2021

Cite: Pop TL. Diagnostic approach to abdominal pain in school-age children. The Journal of School and University Medicine 2021;VIII(1):19-26 
Journal of School and University Medicine

Table 1. The most common causes of acute abdominal pain in school-age children and adolescents. [1,2]

\begin{tabular}{lll}
\hline Age & Common causes & Age-specific causes \\
\hline Schoolchild & Acute appendicitis & Functional abdominal pain \\
(5-11 years) & Intestinal obstruction & Abdominal migraine \\
& Constipation & Intestinal volvulus \\
& Food poisoning & Henoch Schonlein Purpura \\
& Acute gastritis & Lead poisoning \\
\cline { 3 - 3 } Adolescents & Acute gastroenterocolitis & Functional abdominal pain \\
(12-18 years old) & Mesenteric lymphadenitis & Irritable bowel syndrome \\
& Pancreatitis & Inflammatory bowel disease \\
& Biliary pathology & Premenstrual syndrome \\
& Abdominal trauma & Ectopic pregnancy, pregnancy-related disorders \\
& Acute respiratory tract infections & Ovarian or testicular torsion \\
& Urinary tract infection & Pelvic inflammatory disease. Sexually Transmitted \\
& Hemolytic uremic syndrome & Infections (Chlamydia trachomatis, Neisseria gonorrhoeae) \\
\hline
\end{tabular}

The assessment of school-age children with abdominal pain should be based on a thorough anamnesis, followed by a complete objective examination. The child's age may direct the physician to certain more common etiologies (see Table 1). The assessment of pain should consider the location and irradiation, time of onset, nature, severity, aggravating, or pain-reducing factors. The history of medication is important, as well as the associated symptoms. Thus bilious vomiting, constipation, diarrhea, bloody stools, abdominal distension, or fever are important elements that guide the diagnosis. Also, associated symptoms such as cough, dyspnea, fever, pharyngeal pain may be caused by a respiratory infection, while urinary symptoms (dysuria, hematuria, frequent urination) are associated with urinary tract infection. In girls at puberty, an evaluation of the manifestations related to menstruation, sexual activity, and contraception is also necessary. Dehydration, weight loss, vomiting, polyuria, and polydipsia indicate possible abdominal pain in the context of diabetic ketoacidosis. Arthralgia, rashes and dark urine may suggest a Henoch-Schonlein purpura. A history of abdominal trauma may explain the cause of abdominal pain. [1,2]

The doctor must assess the general condition of the child, the level of activity, the food and fluid intake. Assessment of vital signs should include fever (infection or inflammation), tachycardia (sepsis, shock, intestinal obstruction, peritonitis), tachypnea (pneumonia, diabetes acidosis - Kussmaul respiration, uremia, severe dehydration), hypotension (hypovolemia in severe dehydration or losses in the third space in intestinal obstructions or perforations) or hypertension (Henoch-Schonlein purpura, hemolytic uremic syndrome). [1-3] Examination of the abdomen should look for points of maximum pain, any signs of abdominal trauma, palpation of abdominal formations, listening to abdominal noises (ileus or intestinal obstruction). The genito-pelvic examination is essential for possible inguinal hernias, testicular torsion, localized pelvic formations, anal fissures. The clinical exam may reveal jaundice, pallor, purpuric rash, pulmonary stethacoustic changes, or even pharyngeal and tonsillar congestion. [3] The Murphy sign may also be present in acute cholecystitis, and the Cullen and Gray Turner sign in abdominal bleeding. [2]

Laboratory investigations should be adapted to the symptoms and the changes highlighted during the objective examination. Complete blood count (leukocytes, hemoglobin), markers of inflammation (C-reactive protein, erythrocyte sedimentation rate), liver tests (transaminases, bilirubin), amylase, lipase, urine summary examination (leukocyturia, hematuria, glucose, ketones), stool examination (highlighting blood in the stool) and copro-parasitological tests should be taken into account.

Imaging methods include abdominal radiography (especially where the cause of the pain may be an indication for surgery, such as intestinal obstruction or perforation), chest radiography (to rule out pneumonia), abdominal ultrasound, and abdominal computed tomography (CT). 
Table 2. Indications for surgical consultation in acute abdominal pain in children and adolescents [1-3]

- Severe pain or pain that is worsening and is associated with altered general condition

- Biliary or fecal vomiting, hematemesis

- Bloody diarrhea

- Fever

- Absence of intestinal noises

- Abdominal guarding

- Abdominal distension, tympanism

- Signs of acute abdominal bleeding

- Signs of abdominal trauma

- Suspicion of a surgical cause

- Abdominal pain without obvious etiology

Recurrent abdominal pain is one of the most common causes of visiting the pediatrician or pediatric gastroenterologist [4] and affects about $12-15 \%$ of the pediatric population. Only in $5-10 \%$ of cases an organic cause responsible for abdominal pain is identified. [5] These recurrent pains rarely occur in children under four years of age, being more common in school-age and adolescence. For a better understanding of the terms used concerning abdominal pain, here are the different definitions:

- Recurrent abdominal pain: at least three episodes of pain over three months, pain severe enough to affect the child's normal activities (Apley criteria, described in 1958, [6]);

- Chronic abdominal pain: episodic pain, which lasts at least a month, but more often it is more than three months;

- Functional, psychogenic abdominal pain: abdominal pain in the absence of anatomical changes, inflammation, or proven organic lesions;

- Nonspecific abdominal pain: episodic abdominal pain in a child in whom the clinical examination is normal and there is no obvious association with acute gastroenteritis. [7]

The etiology of recurrent/chronic abdominal pain. The possible causes of recurrent abdominal pain in children and adolescents are numerous, from organic to functional causes. Although $73-89 \%$ of children with recurrent abdominal pain may be classified as having a functional gastrointestinal pathology $[8,9]$, the organic causes responsible for these manifestations must be considered (Table 3 ).

Table 3. Organic causes of recurrent abdominal pain in school-age children [7]

- Digestive: celiac disease, gastritis and peptic ulcer, esophagitis (eosinophilic, erosive), gastroesophageal reflux disease, hiatal hernia, Helicobacter pylori (Hp) infection, biliary pathology, gastro-enterocolitis, inflammatory bowel disease, pancreatitis;

- Genito-urinary: endometriosis, premenstrual syndrome, pathology related to menstruation/ ovulation, ovarian cyst, pelvic inflammatory disease, testicular pain, urinary stones;

- Metabolic: adrenal crisis, diabetic ketoacidosis, porphyria;

- Musculoskeletal or neurological;

- Others: recurrent Mediterranean fever, food allergy, lead poisoning, lymphoma, tumors or peritoneal abscesses, pneumonia, vasculitis, nodular polyarteritis.

The pediatrician and the school doctor must consider the alarm signs that can be identified during the anamnesis or the objective examination of a child with abdominal pain. These alarm signs can direct the diagnosis to an organic pathology and require additional evaluation for it. (Table 4). 
Table 4. Clinical warning signs in abdominal pain $[7,10]$

\begin{tabular}{ll}
\hline Anamnesis & Dysphagia, odynophagia \\
Persistent or cyclical biliary vomiting \\
Digestive bleeding (hematemesis or melena) \\
Persistent pain located at a distance from the periumbilical area \\
Nocturnal diarrhea \\
Severe chronic diarrhea \\
Involuntary weight loss \\
Fever of unknown origin \\
Symptoms influence the current activity (learning or playing) \\
Family history of celiac disease, inflammatory bowel disease, or peptic ulcer \\
\hline Clinical examination & Altered stature growth \\
Uveitis \\
Oral lesions \\
Rash, jaundice, or pallor (anemia) \\
Hepatomegaly and/or splenomegaly \\
Arthritis \\
Palpation of an abdominal mass \\
Costo-vertebral or spinal swelling \\
Perianal abnormalities \\
\hline
\end{tabular}

The Rome criteria for functional digestive disorders. In functional abdominal pain, there are no biochemical markers or structural changes that allow an objective diagnosis or the monitoring of these disorders. Thus, it was necessary to establish uniform, easy-to-use criteria for proper assessment and diagnosis. For patients and parents, establishing a clearly defined diagnosis allows a better understanding and acceptance of the diagnosis, despite the impossibility of demonstrating an organic cause. The Rome criteria were first developed in 1990 but were only applicable to adults. In 1999, the Rome II criteria were launched, and criteria were also established for functional gastrointestinal disorders in children. When the criteria were revised in 2006, a distinction was made between functional gastrointestinal disorders in the newborn and infant compared to the older child and adolescent (Rome III criteria). The latest revision took place in 2016 when the Rome IV criteria were published. In the following, we shall detail only the Roma IV criteria for abdominal pain in school-age children and adolescents, including functional dyspepsia, irritable bowel syndrome, abdominal migraine, and functional abdominal pain (not otherwise classified). [10,11] These criteria help a proactive diagnosis of functional gastrointestinal disorders and not as an exclusionary diagnosis. [4]
H2a. Functional dyspepsia is defined by the presence of at least one of the following bothersome symptoms at least four days a month for at least two months before diagnosis:

1. Postprandial fullness.

2. Early satiety.

3. Epigastric pain or burning not associated with defecation

4. After appropriate evaluation, the symptoms cannot be fully explained by another medical condition.

- H2a1. Postprandial distress syndrome includes bothersome postprandial fullness or early satiation, which prevents finishing a regular meal. Supportive features include upper abdominal bloating, postprandial nausea, or excessive belching;

- H2a2. Epigastric pain syndrome which includes bothersome (severe enough to interfere with normal activities) pain or burning localized to the epigastrium. The pain is not generalized or localized to other abdominal or chest regions and is not relieved by defecation or passage of the flatus.

- H2b. Irritable bowel syndrome is defined by the mandatory presence of the following symptoms, at least two months before diagnosis:

1. Abdominal pain at least four days per month, associated with one or more of the following: 
a. Related to defecation;

b. A change in frequency of stool;

c. A change in form (appearance) of stool.

2. In children with abdominal pain and constipation, the pain does not resolve with resolution of constipation (children in whom the pain resolves have functional constipation)

3. After appropriate evaluation, the symptoms cannot be fully explained by another medical condition.

H2c. Abdominal migraine. Must include all of the following, occurring at least twice per month, at least six months before diagnosis:

1. Paroxysmal episodes of intense, acute periumbilical, midline, or diffuse abdominal pain lasting one hour or more (should be the most severe and distressing symptom).

2. Episodes are separated by weeks to months without symptoms.

3. The pain is incapacitating and interferes with normal activities.

4. Stereotypical pattern and symptoms in the individual patient.

5. The pain is associated with two or more of the following:
a. Anorexia;
b. Nausea;
c. Vomiting;
d. Headache;
e. Photophobia;
f. Pallor.

6. After appropriate evaluation, the symptoms cannot be fully explained by another medical condition.

H2d. Functional abdominal pain (not otherwise specified) is defined by the mandatory presence of the following symptoms at least four days a month for at least two months before diagnosis:

1. Episodic or continuous abdominal pain occurring not solely during physiologic events (e.g., eating, menses).

2. Insufficient criteria for irritable bowel syndrome, functional dyspepsia, or abdominal migraine.

3. After appropriate evaluation, the abdominal pain cannot be fully explained by another medical condition.

Epidemiology of functional abdominal pain. About $50 \%$ of children who visit a pediatric gastroenterologist have criteria for at least one of the four functional entities described above. [12] Based on the Rome IV criteria, studies in the United States have shown a prevalence of $16.9 \%$ of these functional disorders. [13] A meta-analysis of the epidemiology of these functional disorders globally showed a prevalence of $13.5 \%$, of which irritable bowel syndrome is the most common (8.8\%). A higher prevalence was found in South America (16.8\%) and Asia (16.5\%) compared to Europe $(10.5 \%)$. The prevalence is higher in girls $(15.9 \%)$ and is associated with anxiety, depression, stress, or traumatic events. [14]

Etiopathogenesis and risk factors in functional abdominal pain. The pathogenesis of functional abdominal pain is based on the interrelationship between changes in visceral sensitivity and altered gastrointestinal motility, resulting from the intervention of multiple factors in a conceptual bio-psycho-social model. These functional disorders with abdominal pain are considered a disorder of the gut-brain interaction, now known as the microbiota-gut-brain axis. [10,15]

The main elements of the etiopathogenesis and risk factors for functional abdominal pain described in the literature:

- Visceral hypersensitivity, central hypervigilance (which causes symptoms such as pain and discomfort);

- Disorders of gastrointestinal motility (causing diarrhea, constipation, nausea, vomiting, bloating);

- Various early life events: digestive intolerance to cow's milk proteins, pyloric stenosis, umbilical hernia, Henoch-Schonlein purpura;

- Psychological factors, stress (separation from the best friend, failure in exams, loss of employment by parents, repeated hospitalizations, abuse), depression, anxiety;

- Inflammation of the intestinal mucosa (there is minimal inflammation in irritable bowel syndrome, eosinophilia in functional dyspepsia);

- Mast cell dysfunction and the role of serotonin (increased in the rectal mucosa in children with irritable bowel syndrome);

- Intestinal microbiota (concerning the birth, diet, use of antibiotics);

- Genetic and environmental factors;

- The role of intestinal infections (onset of irritable bowel syndrome after an episode of acute gastroenterocolitis). [4,10,15] 
The usefulness of diagnostic tests in functional abdominal pain. As can be seen in the Rome IV Criteria, the decision to use diagnostic tests is left to the physician. Frequently, there is a belief that the patient and his parents would not accept a functional diagnosis without laboratory or imaging investigations. Some doctors believe that through education, parents can be made clear of the futility of extensive investigations in children with elements that allow an obvious functional diagnosis. However, the doctor must consider all possible differential diagnoses, warning signs for organic causes and use all necessary diagnostic tests.

Abdominal ultrasound is the most commonly used imaging method for assessing abdominal pain. In functional abdominal pain, it seems to be used mainly to reassure parents rather than to identify a specific etiology. Studies show that abdominal ultrasound does not significantly contribute to the diagnosis of functional abdominal pain. Thus, only in $2-3 \%$ of cases, abdominal ultrasound had a diagnostic role, increasing to $11 \%$ if certain symptoms are associated (jaundice, vomiting, low back pain, urinary symptoms). Therefore, abdominal ultrasound is recommended if children with abdominal pain also show significant clinical changes. [10,12]

Performing digestive endoscopy is an important decision given the expectations of parents and the uncertainty of a specific diagnosis. Certain changes can be identified (such as lymph node hyperplasia, and, histologically, the presence of mast cells and eosinophils) without an important diagnostic value, being described in functional dyspepsia [15]. In a study conducted in the United States on 290 children with chronic abdominal pain, digestive endoscopy had a diagnostic intake in $38 \%$ of cases. The presence of at least 2 warning signs was significant for those with a specific diagnosis. Thus, reflux esophagitis, eosinophilic or erosive esophagitis, as well as a case with celiac disease and $\mathrm{Hp}$ infection, were more frequently identified. Thus, it is recommended that digestive endoscopy be used in children with chronic abdominal pain only in the presence of alarm signs. [10,12]

Regarding the copro-parasitological examination, the Rome IV criteria do not specifically recommend this investigation. Still, studies in some developing countries claim that the local prevalence of parasitic infections would justify this test. In contrast, in developed countries, the prevalence of parasitic infections does not justify such testing. [12]
The recent use of fecal calprotectin as a non-invasive test for assessing intestinal inflammation has shown that at a normal value, digestive endoscopy and histology have no indication for diagnosis. An increased value of fecal calprotectin has a sensitivity of $70 \%$, specificity of $93 \%$, a positive predictive value of $96 \%$, and a negative predictive value of $56 \%$ for organic causes. [16] Thus fecal calprotectin is much increased in inflammatory bowel disease. But it can be positive, with lower values, however, in other situations than in inflammatory bowel disease, such as infections (Giardia lamblia, bacterial dysentery, viral gastroenterocolitis, Hp gastritis), malignancies (colorectal cancer, gastric cancer, intestinal lymphoma), use of drugs (non-steroidal anti-inflammatory drugs, proton pump inhibitors), food allergies, children under 5 years of age or in gastroesophageal reflux disease, cystic fibrosis, celiac disease, diverticular disease, liver cirrhosis. [17]

Fecal lactoferrin, a glycoprotein that binds iron, has recently become more and more indicated in children with abdominal pain, given its increased concentration in stool in intestinal inflammation. However, there are few studies on lactoferrin compared to fecal calprotectin, and the usefulness of lactoferrin in differentiating inflammatory bowel disease and irritable bowel syndrome is not clearly established. Specialists do not recommend using this test to establish a possible organic diagnosis in children with abdominal pain. [12]

As for the screening for celiac disease in children with abdominal pain, some studies show an increased prevalence of celiac disease among patients with irritable bowel syndrome (even four times higher than the general population). Although population studies do not support these increased probabilities, the Rome IV criteria recommend serological screening with anti-transglutaminase $\operatorname{IgA}$ antibodies after assessing serum IgA levels in children with a probable diagnosis of irritable bowel syndrome (especially with diarrhea). [12]. Screening for celiac disease and evaluation of fecal calprotectin are the only cost-effective measures in children with functional abdominal pain without warning signs. [15]

Recently, we are witnessing an increase in indications for testing for $\mathrm{Hp}$ infection in children with abdominal pain using non-invasive evaluation with serological or stool tests. Professional organizations' guidelines do not recommend diagnostic evaluation for $\mathrm{Hp}$ infection in children with functional abdomi- 
nal pain. The "test-and-treat" strategy in Hp infection is not recommended in children. Also, there was no evidence of a relationship between Hp infection and recurrent abdominal pain. Eradication of $\mathrm{Hp}$ infection does not correlate with relief of abdominal pain. [15] Regarding the tests to be used for the diagnosis of $\mathrm{Hp}$ infection, in no case are the fecal antigen test or serological tests to be used. For a definite diagnosis, it is recommended to perform upper digestive endoscopy. Given the above, The European Society for Paediatric Gastroenterology Hepatology and Nutrition (ESPGHAN) does not recommend upper gastrointestinal endoscopy in children with functional gastrointestinal disorders in the absence of warning signs or with normal fecal calprotectin. The decision to use these diagnostic methods is individualized. [12]

Prognosis in case of functional abdominal pain. Functional abdominal pain has a chronic evolution, with an average duration of 7.5 months. A study that included 1331 children with functional abdominal pain showed the persistence of symptoms in $29.1 \%$ of them even after 5 years. [18] Another study, including 392 children, showed that $41 \%$ had criteria for functional abdominal pain even after 9 years of follow-up. [19] This functional pathology can influence the child's school performance and social relationships: he is retired, does not participate in sports or other activities with colleagues his age. Children may have anxiety, depression, extra-intestinal manifestations (headache, dizziness, asthenia) affecting the quality of life in adolescence and adulthood. Risk factors for functional disorders with abdominal pain in children include anxiety, somatization, and maternal depression. [10,20] Parent support is essential; they should not overestimate the symptoms, and they should be educated to give a consistent and precise response to the pain. It is necessary to explain the role of stress, anxiety, and depression in maintaining symptoms. [20]

Adults with irritable bowel syndrome most likely had functional symptoms in childhood. Still, studies have not clearly shown that persistent abdominal pain in childhood can predict adult abdominal pain. [20] Between 7 and $40 \%$ of children with functional abdominal pain will have problems at adult age, more often those with severe forms or those with a family history of irritable bowel syndrome, functional abdominal pain, depression, or anxiety. [10.21]

Conclusions. Abdominal pain is a symptom with very different etiologies in children and adoles- cents, being a common cause to see a pediatrician or school doctor. The correct diagnosis requires a rigorous semiological approach, often necessitating extensive examinations. The Rome criteria allow the correct diagnostic classification of functional pathology. Thus, this needs to be known by the school doctor who comes in contact with children who often have a functional disease. A psychosomatic approach is often necessary in these cases, but the alarm signs that may indicate an organic etiology of these abdominal pains should not be forgotten.

\section{References:}

1. Reust CE, Williams A. Acute abdominal pain in children. Am Fam Physician. 2016;93(10):830-836

2. Leung AKC, Sigalet DL. Acute abdominal pain in children. Am Fam Physician. 2003;67:2321-6

3. Iyer R, Nallasamy K. Child with abdominal pain. Indian J Pediatr. 2018;85(1):71-76

4. Zeiter DK. Abdominal pain in children. From the eternal city to the examination room. Pediatr Clin N Am. 2017;64:525-541

5. Romano C, Porcaro F. Current issues in the management of pediatric functional pain. Rev Recent Clin Trials. 2014;9(1):13-20

6. Apley J, Naish N. Recurrent abdominal pains: a field survey of 1,000 school children. Arch Dis Child. 1958;33(168):165-70

7. Reust CE, Williams A. Reccurent abdominal pain in children. Am Fam Physician. 2018;97(12):785-793

8. Walker LS, Lipani TA, Greene JW, Caines K, Stutts J, Polk DB, Caplan A, Rasquin-Weber A. Recurrent abdominal pain: symptom subtypes based on the Rome II Criteria for pediatric functional gastrointestinal disorders. J Pediatr Gastroenterol Nutr. 2004;38(2):187-91.

9. Schurman JV, Friesen CA, Danda CE, Andre L, Welchert E, Lavenbarg T, Cocjin JT, Hyman PE. Diagnosing functional abdominal pain with the Rome II criteria: parent, child, and clinician agreement. J Pediatr Gastroenterol Nutr. 2005;41(3):291-5

10. Korterink J, Devanarayana NM, Rajindrajith S, Vlieger A, Benninga M. Childhood functional abdiminal pain: mechanism and management. Nat. Rev. Gastroenterol. Hepatol. 2015;12:159-171

11. Koppen IJN, Nurko S, Saps M, DiLorenzo C, Benninga MA. The pediatric Rome IV criteria: what's new? Expert Review of Gastroenterology \& Hepatology. 2017;11(3):193-201

12. Llanos-Chea A, Saps M. Utility of diagnostic tests in children with functional abdominal pain disorders. Gastroenterology \& Hepatology 2019;15(8):414-422

13. Robin SG, Keller C, Zwiener R, Hyman PE, Nurko S, Saps M, Di Lorenzo C, Shulman RJ, Hyams JS, Palsson O, van Tilburg MAL. Prevalence of Pediatric Functional Gastrointestinal Disorders Utilizing the Rome IV Criteria. J Pediatr. 2018;195:134-139.

14. Korterink JJ, Diederen K, Benninga MA, Tabbers MM. Epidemiology of pediatric functional abdominal pain disorders: a meta-analysis. PLoS One. 2015;10(5):e0126982 
15. Thapar N, Benninga M, Crowell MD, DiLorenzo C, Mack I, Nurko S, Saps M, Shulman RJ, Szajewska H, van Tilburg MAL, Enck P. Paediatric functional abdominal pain disorders. Nat. Rev. Disease Primers. 2020;6:89

16. Carroccio A, Iacono G, Cottone M, Di Prima L, Cartabellotta F, Cavataio F, Scalici C, Montalto G, Di Fede G, Rini G, Notarbartolo A, Averna MR. Diagnostic accuracy of fecal calprotectin assay in distinguishing organic causes of chronic diarrhea from irritable bowel syndrome: a prospective study in adults and children. Clin Chem. 2003;49(6 Pt 1):861-7

17. van Rheenen PF, Van de Vijver E, Fidler V. Faecal calprotectin for screening of patients with suspected inflammatory bowel disease: diagnostic meta-analysis. BMJ. 2010;341:c3369.

18. Gieteling MJ, Bierma-Zeinstra SM, Passchier J, Berger MY. Prognosis of chronic or recurrent abdominal pain in children. J Pediatr Gastroenterol Nutr. 2008;47(3):316-26
19. Horst S, Shelby G, Anderson J, Acra S, Polk DB, Saville BR, Garber J, Walker LS. Predicting persistence of functional abdominal pain from childhood into young adulthood. Clin Gastroenterol Hepatol. 2014;12(12):2026-32.

20. McClellan N, Ahlawat R. Functional Abdominal Pain In Children. 2020 Jun 29. In: StatPearls [Internet]. Treasure Island (FL): StatPearls Publishing; 2021 Jan

21. Hotopf M, Carr S, Mayou R, Wadsworth M, Wessely S. Why do children have chronic abdominal pain, and what happens to them when they grow up? Population based cohort study. BMJ. 1998;316(7139):1196-200 